\title{
Effect of soil physico-chemical properties and plant type on bacterial diversity in semi- arid parts in central Sudan. Part ii. Sharq El-neel region, Khartoum state
}

\author{
Hayat Ibrahim Hassan, Hatil Hashim El-Kamali* \\ Department of Botany, Faculty of Science and Technology, Omdurman Islamic University, Omdurman, Sudan \\ *Corresponding author E-mail:hatilhashim@gmail.com
}

\begin{abstract}
Total viable counts of bacteria and bacterial diversity of the different soil samples from three different localities in Sharq EL-Neel region: Soba, AL-Aelafoon and Um Dawan Ban sub-regions were carried out. Soil physical and chemical characteristics (pH, EC,SP,solublecations: $\mathrm{Na}, \mathrm{K}, \mathrm{Ca}, \mathrm{Mg}$ and anion $\mathrm{P}$, organic carbon, total nitrogen and soil texture)in each studied sub-regions were measured. Qualitative analysis of microorganisms isolated from the studied soil samples reveal a total of thirteendifferent species of bacteria, of which two are unidentified. The ten species are classified under Bacillus genus. In Sharq EL-Neel regionsoil samples, total bacterial counts ranged from $9.5 \times 10^{4} \mathrm{cfu} \mathrm{g}^{-1}$ to $1 \times 10^{3}$ with a mean of $4 \times 10^{3} \mathrm{cfu} \mathrm{g}^{-1}$. The quantitative data on microbial population recorded in the present study was analysed using two diversity indices. High Shannon-Weiner diversity Index value for bacteria was obtained in ALAelfoon sub-region (1.79361), whereas high Simpson's index value was obtained in Um Dawan Ban sub-region (2.80).ActinomycesActinomyces spp. and Streptomyces spp. Where the most abundant microorganisms identified in the three subregions. Total bacterial count in Soba soil was positively correlated with $\mathrm{pH}(\mathrm{r}=0.0194)$ and sand $(\mathrm{r}=0.3205)$; the total bacterial count in AL-Aelafoon soilwas positively correlated with EC ( $r=0.1062)$, clay $(r=0.3816)$, silt $(r=0.1936), S P(r=0.9302), K(r=0.6252), C a(r=$ $0.0015)$ and $\mathrm{Mg}(\mathrm{r}=0.1556)$, whereas the total bacterial count in Um Dawan Ban soil was positively correlated with clay $(\mathrm{r}=0.2614)$, silt $(\mathrm{r}=0.0216), \mathrm{SP}(\mathrm{r}=0.565), \mathrm{K}(\mathrm{r}=0.9645), \mathrm{P}(\mathrm{r}=0.0197), \mathrm{Ca}(\mathrm{r}=0.7377), \mathrm{Mg}(\mathrm{r}=0.0267), \mathrm{N}(\mathrm{r}=0.5215)$ and O.C $(\mathrm{r}=0.3214)$. There were obvious differences in correlation coefficients among the selected criteria (46\% from the total number of correlation coefficients were positively correlated between bacterial counts and soil physico-chemical properties whereas $54 \%$ from the total number were positively correlated between plant type and bacterial counts).
\end{abstract}

Keywords: Microbial Diversity; Physico-Chemical Properties; Soils; Semi-Arid Zone; Sharq EL-Neel Region; Central Sudan.

\section{Introduction}

Continuing our research works on the relationships between soil physico-chemical properties,plants and soil microorganism's populations in semi-arid parts in Sudan [1], we have study correlationsbetween theseparameters in Sharq EL-Neel region, Khartoum State, Central Sudan.

Soil is a complex habitat, inhabited by a large number of different organisms. Among these, bacteria and fungi are the most important since they are responsible for the vast bulk of decomposition, and also make up the largest part of the biomass in soil. Many of the essential transformations in the nitrogen, sulphur, phosphorus and other element cycles are mediated by microbes.Bacteria are the most abundant microorganism group in soil and can attain concentrations of more than $10^{8}$ cells per gram of soil,or $10^{11}$ per gram organic material [2]. The activity of soil organisms can be divided into four functions: (1) regulation of organic matter turnover and nutrient cycling,(2) biological degradation, (3) maintenance of soil structure,and (4) interaction with plants.

The main factors contributing to the soil environment are: (1) soil texture and structure (2) nutrient status (3) soil pH (4) moisture and temperature (5) surface plants (6) Inputs and (7) compaction. Different soil environments will support different types and num- bers of microorganisms. Different plant residues contain varying quantities and availability of carbon and nutrients. This influences the soil biological activity.

The objectives of this study were: (i) to obtain a better understanding of the correlations between microbial population and physicochemical properties of different soil types in the study area. (ii) to study how plant type and soil type affects the microbial diversity and abundance. (iii) to explain the differences between the tested habitats.

\section{Materials and methods}

\section{1. Study site description and soil sampling}

Soils were collected from three different sub-regions (Soba, ALaelafoon and Um Dawan Ban sub-regions in the Khartoum State,in arid/semi-arid parts in Central Sudan. Soil samples were collected from $0-5 \mathrm{cmand} 5-15 \mathrm{~cm}$ depths and kept in plastic bag. After collection, soil samples were brought to the laboratory and separated into two sub samples; one for bacteriological analysis that was kept in a refrigerator and the other one for the analysis of soil physico-chemical properties. Soil sampling was done in December, 2011. 


\subsection{Bacteriological analysis}

Nutrient agar medium was used for the enumeration of bacteria present in soil samples [3]. The $\mathrm{pH}$ was adjusted before addition of agar and sterilization. Serial dilution plate technique was used for the isolation of microorganism. One gramsoil sample was diluted (1: 100) with $100 \mathrm{ml}$ distilled water in a sterile conical flask and shaken well. One $\mathrm{ml}$ of this suspension was transferred to $9 \mathrm{ml}$ of sterile water for tenfold (1: 10) dilution and by following serial dilution further diluted up to $10^{5}$ times. Plating in duplicate plates was made for each diluted sample. One $\mathrm{ml}$ of each of the diluted sample was taken in a sterilized petri dish by pipette. Then, molten agar medium was poured and mixed thoroughly by rotating the petri dish, first in one direction and then in the opposite direction.After setting the medium, the plates were inverted and incubated at $37^{\circ} \mathrm{C}$ for $48 \mathrm{~h}$ in an incubator then, the plates having well discrete colonies were selected for counting. The selected plates were placed on a colony counter (Digital colony counter, DC8OSK1000086, Kayagaki, Japan) to count the number of colonies.

\subsubsection{Tests}

Motility test was determined according to Cruickshank et al, 1975 [4].Catalase Catalase test Oxidation-Fermentation test (O/F), Oxidase test,Sugar fermentation test,Voges-Proskauer test,Nitrate reductiontest, Indole production test,Urease test,Citrate utilizationwere determined according toBarrow and Feltham 1993 [5] Casein hydrolysis was determined by method described by Williams and Cross, 1971 [6]. .Starch hydrolysis was performed according to Collins et al., 1995 [7]. Total a viable count of bacteria was determined [8].

\subsubsection{Isolation of Streptomyces}

Isolation of Streptomyces was performed by the soil dilution plate technique [9]. In this technique; $1 \mathrm{~g}$ of each soil sample was taken in $9 \mathrm{ml}$ of sterilized distilled water in pre-sterilized test tube. Serial aqueous dilutions $\left(10^{-2}-10^{-7}\right)$ were prepared by transferring $1 \mathrm{ml}$ of the soil suspension into $9 \mathrm{ml}$ of sterilized distilled water in sterilized test tubes. Different aqueous dilutions $\left(10^{-4}-10^{-6}\right)$ of the soil suspensions were applied separately into sterilized Petri-dishes and $20 \mathrm{ml}$ of Starch-Casein Agar salt medium, $\mathrm{SCKNO}_{3}$, was added, mixed thoroughly and the plates were incubated at $28^{\circ} \mathrm{C}$ for 7 14 days. $\mathrm{SCKNO}_{3}$ medium was prepared by dissolving $10 \mathrm{~g}$ soluble starch, $2 \mathrm{~g}$ dipotassium hydrogen ortho-phosphate, $2 \mathrm{~g}$ potassium nitrate, $2 \mathrm{~g}$ sodium chloride, $4 \mathrm{~g}$ casein, $0.05 \mathrm{~g}$ hydrated magnesium sulphate, $0.1 \mathrm{~g}$ calcium carbonate; $0.01 \mathrm{~g}$ hydrated ferric sulphate, $15 \mathrm{~g}$ agar in one liter of distilled water. The medium was sterilized by autoclaving at $121^{\circ} \mathrm{C}$ for 15 minutes. Colonies characteristic of Streptomycetaceae (rough, chalky, powdery and with earth odour) that appeared on the incubated plates were selected, repeatedly sub-cultured for purification and stored at $4^{\circ} \mathrm{C}$ onto slants of $\mathrm{SCKNO}_{3}$ medium until further examinations.

\subsection{Analysis of soil physico-chemical properties}

The $\mathrm{pH}$ of the soil was measured in a soil water suspension (1:2, soil:water). The electrical conductivity (EC) analysis was measured in the saturated extract. $\mathrm{Na}+$ and $\mathrm{K}+$ were determined photometrically. The exchangeable cations $\left(\mathrm{Ca}^{++}\right.$and $\left.\mathrm{Mg}^{++}\right)$were determined by Atomic Absorption Spectrophotometer (AAS, Perkin-Elmer, 047-1705.Saturated percentage (SP) were also determined [10]. Organic carbon content of the soil was determined by Wakely and Black method(Cited by Moghimi et al., [11]. Total nitrogen (\%) was determined by Kjeldahl method following extraction from $2 \mathrm{~g}$ soil with conc. $\mathrm{H}_{2} \mathrm{SO}_{4}$. The particle size analysis was carried out by the Pipette method(Cited by Moghimi et al., 2013 [11]

Once the percentage of sand, silt, and clay is measured, the soil may be assigned a textural class using the Table of textural soil types (Cited by Subrahmanyam and Sambamurty [12].

\subsection{Bacterial diversity measures}

1/ Shannon-Weiner Biodiversity Index:

Species diversity $(\mathrm{H})=-\left(\mathrm{P}_{\mathrm{i}}\right)\left(\log _{2} \mathrm{P}_{\mathrm{i}}\right)$

Where: $\mathrm{P}=$ the proportion of all individuals in the sample which belongs the species $i$.

2/ Simpson Index: $\mathrm{D}=1-\sum \mathrm{S}\left(\mathrm{P}_{\mathrm{i}}\right)^{2}$

$$
\mathrm{i}=1
$$

Where: $\mathrm{D}$ is theindex number; $\mathrm{S}=$ the total number of species; $\mathrm{P}=$ the proportion of all individuals in the sample which belongs to species i

(Cited by Subrahmanyam and Sambamurty [12].

\subsection{Statistical analysis}

Correlation coefficient was performed in order to detect the relationships between soil physic-chemical parameters and microbial populations [13].

\section{Results and discussion}

\subsection{The soil characteristics in Shaq EL-Neelregion}

The results concerning soil physical and chemical characteristics ( $\mathrm{pH}, \mathrm{EC}, \mathrm{SP}$, soluble cations: $\mathrm{Na}, \mathrm{K}, \mathrm{Ca}, \mathrm{Mg}$ and anion $\mathrm{P}$, organic carbon, total nitrogen and soil texture) in three differentstudied sub-regions are presented in Tables 1-3.

\begin{tabular}{|c|c|c|c|c|c|c|c|c|c|c|c|c|c|c|c|c|}
\hline $\begin{array}{l}\text { Sample } \\
\text { No. }\end{array}$ & $\begin{array}{l}\text { Soil } \\
\text { Depth }\end{array}$ & $\begin{array}{l}\text { Bacterial } \\
\text { count } \\
(\mathrm{CFU} / \mathrm{g})\end{array}$ & $\mathrm{pH}$ & $\mathrm{EC}$ & $\mathrm{N} \%$ & O.C \% & SP & $\mathrm{Na}$ & $\mathrm{K}$ & $\mathrm{P}$ & $\mathrm{Ca}$ & $\mathrm{Mg}$ & $\begin{array}{l}\text { Clay } \\
\%\end{array}$ & $\begin{array}{l}\text { Silt } \\
\%\end{array}$ & Sand $\%$ & $\begin{array}{l}\text { Textural } \\
\text { Soil } \\
\text { Types } \\
\end{array}$ \\
\hline SO1 & $0-5$ & $1 \times 10^{4}$ & 7.71 & 0.53 & 0.056 & 0.47 & 26.3 & 3.019 & 0.076 & 1.482 & 2 & 0.75 & 30 & 17 & 53 & $\begin{array}{l}\text { Sandy } \\
\text { clay loam }\end{array}$ \\
\hline $\mathrm{SO} 2$ & $5-15$ & $5 \times 10^{3}$ & 7.63 & 0.88 & 0.035 & 0.32 & 28.4 & 6.431 & 0.047 & 1.729 & 1.5 & 0.5 & 34 & 25 & 41 & $\begin{array}{l}\text { Clay } \\
\text { loam }\end{array}$ \\
\hline $\mathrm{SO} 3$ & $0-5$ & $1.5 \times 10^{3}$ & 7.70 & 0.99 & 0.105 & 0.86 & 28.8 & 5.676 & 0.195 & 1.032 & 5 & 2.3 & 34 & 33 & 34 & $\begin{array}{l}\text { Clay } \\
\text { loam }\end{array}$ \\
\hline SO5 & $0-5$ & $1.7 \times 10^{4}$ & 7.62 & 0.35 & 0.049 & 0.40 & 28.8 & 1.57 & 0.106 & 1.066 & 2 & 0.75 & 21 & 30 & 49 & Loam \\
\hline SO6 & 5-15 & $1.4 \times 10^{3}$ & 7.71 & 0.35 & 0.070 & 0.46 & 31.8 & 2.959 & 0.057 & 1.100 & 2.5 & 1 & 37 & 22 & 41 & $\begin{array}{l}\text { Clay } \\
\text { loam }\end{array}$ \\
\hline SO7 & $0-5$ & $1.5 \times 10^{5}$ & 7.63 & 0.35 & 0.042 & 0.32 & 25.9 & 1.257 & 0 & 0 & 0 & 0 & 21 & 22 & 56 & $\begin{array}{l}\text { Sandy } \\
\text { clay loam }\end{array}$ \\
\hline SO8 & $5-15$ & $1.8 \times 10^{3}$ & 7.49 & 0.35 & 0.049 & 0.61 & 25.1 & 1.419 & 0.096 & 1.872 & 4 & 1.5 & 19 & 25 & 56 & $\begin{array}{l}\text { Sandy } \\
\text { loam }\end{array}$ \\
\hline $\mathrm{SO} 9$ & $0-5$ & $1.8 \times 10^{3}$ & 7.55 & 0.776 & 0.042 & 0.32 & 25.3 & 1.978 & 0.147 & 1.445 & 4 & 1.75 & 19 & 25 & 56 & $\begin{array}{l}\text { Sandy } \\
\text { loam }\end{array}$ \\
\hline
\end{tabular}


Table 2: Some Soil Physico- Chemical Properties of Different Samples from Sharq EL-Neel Region- AL-Aelafoon Subregion

\begin{tabular}{|c|c|c|c|c|c|c|c|c|c|c|c|c|c|c|c|c|}
\hline $\begin{array}{l}\text { Sample } \\
\text { No. }\end{array}$ & $\begin{array}{l}\text { Soil } \\
\text { Depth }\end{array}$ & $\begin{array}{l}\text { Bacterial } \\
\text { count } \\
(\mathrm{CFU} / \mathrm{g})\end{array}$ & $\mathrm{pH}$ & $\mathrm{EC}$ & $\mathrm{N} \%$ & O.C \% & SP & $\mathrm{Na}$ & $\mathrm{K}$ & $\mathrm{P}$ & $\mathrm{Ca}$ & $\mathrm{Mg}$ & $\begin{array}{l}\text { Clay } \\
\%\end{array}$ & $\begin{array}{l}\text { Silt } \\
\%\end{array}$ & Sand $\%$ & $\begin{array}{l}\text { Textural } \\
\text { Soil } \\
\text { Types }\end{array}$ \\
\hline AL1 & $0-5$ & $1.2 \times 10^{5}$ & 7.66 & 0.788 & 0.046 & 0.48 & 36.4 & 2.630 & 0.109 & 0 & 5 & 1.5 & 24 & 32 & 44 & Loam \\
\hline AL2 & $5-15$ & $1 \times 10^{4}$ & 7.70 & 0.617 & 0.063 & 0.64 & 26.3 & 1.510 & 0.052 & 0.628 & 2.5 & 1 & 28 & 24 & 51 & $\begin{array}{l}\text { clay } \\
\text { loam }\end{array}$ \\
\hline AL3 & $0-5$ & $2.8 \times 10^{3}$ & 7.73 & 0.40 & 0.063 & 0.72 & 22.1 & 0.804 & 0.119 & 0.628 & 4.5 & 1.5 & 22 & 34 & 44 & Loam \\
\hline AL4 & $5-15$ & $1.3 \times 10^{3}$ & 7.69 & 0.37 & 0.042 & 0.63 & 30.1 & 1.963 & 0.039 & 1.031 & 2 & 0.75 & 20 & 27 & 54 & $\begin{array}{l}\text { Sandy } \\
\text { loam }\end{array}$ \\
\hline AL5 & $0-5$ & $1.2 \times 10^{4}$ & 7.73 & 0.40 & 0.042 & 0.64 & 26.3 & 1.522 & 0.112 & 1.273 & 3 & 0.5 & 21 & 35 & 44 & Loam \\
\hline AL6 & $5-15$ & $1 \times 10^{3}$ & 7.73 & 0.32 & 0.035 & 0.45 & 28.0 & 1.522 & 0.036 & 0 & 1.5 & 0.5 & 35 & 37 & 29 & $\begin{array}{l}\text { Clay } \\
\text { loam }\end{array}$ \\
\hline AL7 & $0-5$ & $1 X 10^{3}$ & 7.44 & 0.857 & 0.042 & 0.46 & 31.8 & 4.456 & 0.081 & 0 & 2.5 & 1.5 & 21 & 35 & 44 & Silt loam \\
\hline AL8 & $5-15$ & $1.1 \times 10^{3}$ & 7.51 & 0.356 & 0.035 & 0.23 & 23.4 & 2.413 & 0.026 & 1.342 & 1.5 & 0.5 & 22 & 37 & 41 & Loam \\
\hline AL9 & $0-5$ & $2 \times 10^{5}$ & 7.40 & 0.95 & 0.028 & 0.31 & 38.9 & 3.080 & 0.195 & 0.930 & 4.5 & 2 & 35 & 37 & 29 & $\begin{array}{l}\text { Clay } \\
\text { loam }\end{array}$ \\
\hline AL10 & $5-15$ & $7.5 \times 10^{3}$ & 7.58 & 0.43 & 0.021 & 0.40 & 27.6 & 1.691 & 0.053 & 5.179 & 2 & 0.75 & 30 & 32 & 39 & $\begin{array}{l}\text { Clay } \\
\text { loam }\end{array}$ \\
\hline AL11 & $0-5$ & $1.2 \times 10^{4}$ & 7.61 & 0.35 & 0.028 & 0.64 & 25.5 & 1.268 & 0.077 & 1.135 & 3 & 1 & 25 & 27 & 49 & $\begin{array}{l}\text { Loam } \\
\text { Sandy }\end{array}$ \\
\hline AL12 & $5-15$ & $1.6 \times 10^{4}$ & 7.42 & 0.45 & 0.056 & 0.40 & 27.6 & 1.721 & 0.045 & 0.594 & 2.5 & 1 & 24 & 20 & 56 & $\begin{array}{l}\text { clay } \\
\text { loam }\end{array}$ \\
\hline AL13 & $0-5$ & $1.7 \times 10^{4}$ & 7.36 & 2.00 & 0.035 & 0.45 & 25.5 & 4.63 & 0.178 & 0 & 20 & 4 & 35 & 27 & 39 & $\begin{array}{l}\text { Clay } \\
\text { loam }\end{array}$ \\
\hline AL14 & $5-15$ & $7.5 \times 10^{3}$ & 7.69 & 2.434 & 0.035 & 0.46 & 27.5 & 12.05 & 0.104 & 0 & 19 & 4 & 20 & 39 & 41 & Loam \\
\hline
\end{tabular}

Table 3: Some Soil Physico- Chemical Properties of different Samples from Sharq EL-Neel Region- Um Dawan Ban

\begin{tabular}{|c|c|c|c|c|c|c|c|c|c|c|c|c|c|c|c|c|}
\hline $\begin{array}{l}\text { Sample } \\
\text { No. }\end{array}$ & $\begin{array}{l}\text { Soil } \\
\text { Depth }\end{array}$ & $\begin{array}{l}\text { Bacterial } \\
\text { count } \\
(\mathrm{CFU} / \mathrm{g})\end{array}$ & $\mathrm{pH}$ & EC & $\mathrm{N} \%$ & O.C \% & SP & $\mathrm{Na}$ & $\mathrm{K}$ & $\mathrm{P}$ & $\mathrm{Ca}$ & $\mathrm{Mg}$ & $\begin{array}{l}\text { Clay } \\
\%\end{array}$ & $\begin{array}{l}\text { Silt } \\
\%\end{array}$ & Sand \% & $\begin{array}{l}\text { Textural } \\
\text { Soil } \\
\text { Types }\end{array}$ \\
\hline UD1 & $0-5$ & $4 \times 10^{5}$ & 7.49 & 2.30 & 0.042 & 0.45 & 37.7 & 7.043 & 0.188 & 0 & 23 & 4 & 24 & 38 & 39 & $\begin{array}{l}\text { Loam } \\
\text { Sandy }\end{array}$ \\
\hline UD2 & $5-15$ & $4 \times 10^{4}$ & 7.48 & 4.00 & 0.038 & 0.46 & 30.9 & 31.40 & 0.070 & 1.623 & 24 & 4 & 21 & 23 & 55 & $\begin{array}{l}\text { clay } \\
\text { loam }\end{array}$ \\
\hline UD3 & $0-5$ & $3 \times 10^{5}$ & 7.70 & 0.44 & 0.056 & 0.61 & 31.4 & 1.956 & 0.154 & 0 & 2.5 & 0.5 & 19 & 13 & 69 & $\begin{array}{l}\text { Sandy } \\
\text { loam } \\
\text { Sandy }\end{array}$ \\
\hline UD4 & $5-15$ & $9.5 \times 10^{4}$ & 8.09 & 0.94 & 0.056 & 0.64 & 25.9 & 6.891 & 0.07 & 0 & 2.5 & 0.5 & 31 & 15 & 54 & $\begin{array}{l}\text { clay } \\
\text { loam }\end{array}$ \\
\hline UD5 & $0-5$ & $6.5 \times 10^{5}$ & 7.81 & 1.12 & 0.07 & 0.65 & 30.9 & 5.405 & 0.224 & 1.412 & 5 & 1.50 & 35 & 19 & 46 & $\begin{array}{l}\text { clay } \\
\text { loam }\end{array}$ \\
\hline UD6 & $5-15$ & $3.5 \times 10^{4}$ & 7.82 & 0.94 & 0.056 & 0.54 & 23.9 & 4.438 & 0.097 & 0.896 & 4 & 1 & 31 & 28 & 41 & $\begin{array}{l}\text { Clay } \\
\text { loam }\end{array}$ \\
\hline
\end{tabular}

\subsubsection{Soba sub-region}

The soil of this sub-region is predominantly sandy clay loam. The $\mathrm{pH}$ of soil samples ranged from 7.49 to 7.71 . The EC values varied from $0.35-0.99 \mathrm{mmohs} / \mathrm{cm}$. The total nitrogen was in range $0.028-0.105$. Organic carbon range between 0.32 and $0.86 \%$. $\mathrm{C}: \mathrm{N}$ ratio range between $8: 1$ and 13:1. The SP ranged from $25.1-$ $31.8 \%$. Sodium contents ranges between 1.257 and $6.431 \mathrm{Meq} / \mathrm{L}$. As for $\mathrm{K}$ it varies between 0.0 and $0.195 \mathrm{Meq} / \mathrm{L}$. Calcium contents was found to vary between $0.0-5.0 \mathrm{Meq} / \mathrm{L}$. Magnesium contents was found to vary between 0.0 and $2.3 \mathrm{Meq} / \mathrm{L} . \mathrm{P}$ contents ranged between 0.0 and $1.872 \mathrm{ppm}$.

\subsubsection{Al-aelafoon sub-region}

The soil of this sub-region is predominantlyclay loam. The $\mathrm{pH}$ of soil samples ranged from 7.36 to 7.73 . The EC values varied from $0.32-2.434 \mathrm{mmohs} / \mathrm{cm}$. The total nitrogen was in range $0.021-$ 0.063 . Organic carbon range between 0.23 and $0.72 \%$. C:N ratio range between 7:1 and 23:1.The SP ranged from $22.1-38.9 \%$. Sodium contents ranges between 0.804 and $4.63 \mathrm{Meq} / \mathrm{L}$. As for K it varies between 0.026 and $0.195 \mathrm{Meq} / \mathrm{L}$. Calcium contents was found to vary between $1.5-20 \mathrm{Meq} / \mathrm{L}$. Magnesium contents was found to vary between 0.5 and $4.0 \mathrm{Meq} / \mathrm{L}$.P contents ranged between 0.0 and $5.179 \mathrm{ppm}$.

\subsubsection{Um dawan ban sub-region}

The soil of this sub-region is predominantlysandy clay loam. The $\mathrm{pH}$ of soil samples ranged from 8.00 to 7.48. The EC values varied from $0.44-4.0 \mathrm{mmohs} / \mathrm{cm}$. The total nitrogen was in range $0.038-0.07$. Organic carbon range between 0.45 and $0.65 \%$.C: $\mathrm{N}$ ratio range between 9:1 and 12:1. The SP ranged from 23.9-37.7 $\%$. Sodium contents ranges between 1.956 and $31.043 \mathrm{Meq} / \mathrm{L}$. As for $\mathrm{K}$ it varies between 0.070 and $0.224 \mathrm{Meq} / \mathrm{L}$. Calcium contents was found to vary between $2.5-23 \mathrm{Meq} / \mathrm{L}$. Magnesium contents was found to vary between 0.5 and $4.0 \mathrm{Meq} / \mathrm{L} . \mathrm{P}$ contents ranged between 0.0 and $1.623 \mathrm{ppm}$.

From the 30 collected soils, five different textural soil classes (sandy clay loam, clay loam, loam, sandy loam and silt loam) were detected (Tables 1 -3). The data of soil $\mathrm{pH}$ values range between 8.09 (in Um Dawan Ban) to 7.36 (in AL-Aelafoon) among different soil textures. Sandy clay loam (in Soba and Um Dawan Ban)and clay loam (in Soba and AL-Aelafoon) soils showed highest bacterial populations.

\subsection{Bacterial diversity and total counts of different soil samples from study region:}

The diversity of soil microorganisms of the study habitat is presented in Table 4. Thirteen organisms were isolated from collected soil samples; Actinomyces spp., Streptomyces spp., Bacillus lentus , Bacillus badius, Bacillus pantothenticus, Bacillus mycoides, Bacillus alvei, Bacillus circulans, Bacillus subtilis, Bacillus cere- 
us, Bacillus marcerans, Bacillus thruingiensis,Micrococcus varians. Actinomycetes spp. have highest frequency in the three studied sub- regions and next are Streptomyces spp.

Bacteria species like Bacillus lentus, B.circulans, B. cereus, B. mycoides, B. badius, B. thuringensis, Actinomyces spp. and Strep- tomyces spp. were found in the three habitats. These species are habitat generalists and are well adapted to change in environmental conditions.

Table 4:Total Bacterial Count of Different Soil Samples from Sharq EL-Neel Region

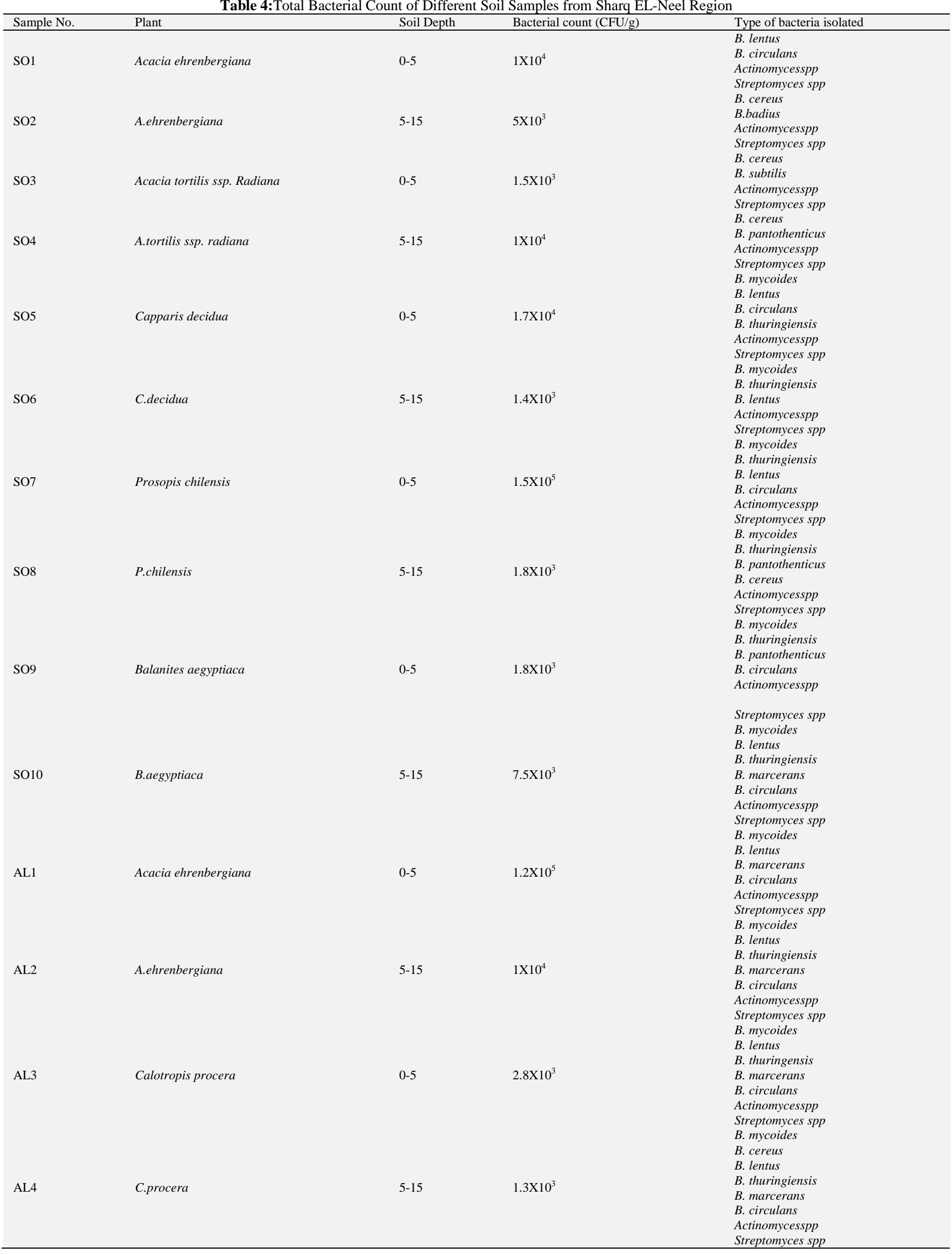


AL5

Balanites aegyptiaca

$0-5$

$1.2 \times 10^{4}$

AL6

B.aegyptiaca

AL7

Capparis deciduas

$0-5$

$1 \times 10^{3}$

AL8

C.decidua

$5-15$

0-5

Acacia tortilis ssp. spirocarpa

A.tortilis ssp. Spirocarpa

$5-15$

$0-5$

Ziziphus spina- Christ

Z. spina-Christi

Prosopis chilensi

P.chilensi

AL14

UD1

Acacia tortilis ssp. tortilis

$0-5$

5-15

A.tortilis ssp. tortilis

Acacia tortilis ssp. radiana

A.tortilis ssp. radiana

Acacia ehrenbergiana

$0-5$

$5-15$
B. mycoides

B. cereus

Actinomycesspp

Streptomyces spp

B. mycoides

$B$. thuringiensis

B. marcerans

Actinomycesspp

Streptomyces spp

B. lentus

B. thuringiensis

B. cereus

B. mycoides

Actinomycesspp

Streptomyces spp

B.badius

B. lentus

B. mycoides

B. marcerans

Actinomycesspp

Streptomyces spp

B. mycoides

B. lentus

B. circulans

B. thuringiensis

B. marcerans

Actinomycesspp

Streptomyces spp

B. thuringiensis

B.cereus

B.mycoides

Actinomycesspp

Streptomyces spp

B. thuringiensis

B.cereus

B.mycoides

Actinomycesspp

Streptomyces spp

$B$. thuringiensis

B.cereus

B.mycoides

Actinomycesspp

Streptomyces spp

$B$. thuringiensis

B.cereus

B.mycoides

Actinomycesspp

Streptomyces spp

$B$. thuringiensis

B.cereus

B.mycoides

Actinomycesspp

Streptomyces spp

$B$. thuringiensis

B.cereus

B.mycoides

Actinomycesspp

Streptomyces spp

$B$. mycoides

B. circulans

B. lentus

Actinomyces spp.

Streptomyces spp

B. mycoides

B. circulans

B.alvei

B. lentus

Actinomyces spp.

Streptomyces spp.

B. cereus

B. pantothenticus

B. mycoides

Micrococcus varians

Actinomycesspp

Streptomyces spp

B. cereus

B. pantothenticus

B.alvei

B. lentus

B. mycoides

Micrococcus varian

B.badius

Actinomycesspp

Streptomyces spp 
Our results showed that microbial population was different in soil under different plant covers, soil types and depths. The total number of isolated bacteria varied in different samples of studied soils. Among several factors affecting microbial population and activity, moisture, temperature, nutrients and soil depth are important factors.

Bacterial count tends to decrease with increase in soil depth. Decrease in the bacterial count with increasing soil depth could be related to the organic carbon content of the soil as nutrients are declining with the increase in soil depth. The higher bacterial count at the surface layer might be due to the presence of litters, twigs, herbs and tree canopy which render a moist environment in the soil and favor high microbial activity and hence high microbial populations [14].

\subsection{Quantitative data on microbial populations using diversity indices}

The quantitative data on microbial population recorded in the present study was analysed using two diversity indices. High Shannon-Weiner diversity Index value for bacteria was obtained in AL-Aelfoon sub-region (1.79361), whereas high Simpson index value was obtained in Um Dawan Ban sub-region (2.80). Table 5.

\begin{tabular}{lll}
\multicolumn{2}{c}{ Table 5: Diversity of Microorganisms in the Study Area } \\
\hline \multirow{2}{*}{ Sub-region } & Shannon-Weiner Diversity & $\begin{array}{l}\text { SimpsonDiversity } \\
\text { Index }\end{array}$ \\
& Index & 1.78 \\
Soba & 1.3453 & 2.7213 \\
AL-Aelafoon & 1.79361 & 2.80 \\
Um Dawan Ban & 1.5408 & \\
\hline
\end{tabular}

\subsection{The correlation effects between the soils character-} istics on bacterial count:

The correlation effects between the soils parameters on bacterial count were studied (Tables 6 - 8).

\subsubsection{Soba}

Total bacterial count was positively correlated with $\mathrm{pH}(\mathrm{r}=$ $0.0194)$ and sand (0.3205) and negatively correlated with EC, clay, silt, SP, Na, K, P, Ca, Mg, N, and OC. Table 6.

\subsubsection{AL-aelafoon sub-region}

Total bacterial count was positively correlated with EC ( $\mathrm{r}=$ $0.1062)$, clay $(\mathrm{r}=0.3816)$, silt $(\mathrm{r}=0.1936), \mathrm{SP}(\mathrm{r}=0.8302), \mathrm{K}(\mathrm{r}=$ $0.6252), \mathrm{Ca}(\mathrm{r}=0.0015)$, and $\mathrm{Mg}(\mathrm{r}=0.1556)$. Table 7 .

\subsubsection{Um dawan ban sub-region}

Total bacterial count was positively correlated with clay ( $\mathrm{r}=$ $0.2614)$, silt $(\mathrm{r}=0.0216)$, $\mathrm{SP}(\mathrm{r}=0.565), \mathrm{K}(\mathrm{r}=0.9645), \mathrm{P}(\mathrm{r}=$ $0.0197), \mathrm{Ca}(\mathrm{r}=0.7377), \mathrm{Mg}(\mathrm{r}=0.0267), \mathrm{N}(\mathrm{r}=0.5215)$ and $\mathrm{OC}$ $(\mathrm{r}=0.3214)$ and negatively correlated with $\mathrm{pH}, \mathrm{EC}$, sand and $\mathrm{Na}$ Table 8.

All the relationships between the total viable bacterial counts and soil physico-chemical properties or plant types are compiled in Tables $6-8$. There were obvious differences in correlation coefficients among the selected criteria ( $46 \%$ from the total number of correlation coefficients were positively correlated between bacterial counts and soil physico-chemical properties whereas $54 \%$ from the total number were positively correlated between plant type and bacterial counts).
Table 6:Correlation Coefficients of the Physico-Chemical Properties with the Viable Bacterial Count $\left(\mathrm{Cfu} \mathrm{G}^{-1}\right.$ Soil) In Soba Sub-Region

\begin{tabular}{llll}
\hline Soil Physico-chemical Properties & $\mathrm{R}$ & $\mathrm{R}^{2}$ & Correlation \\
\hline $\mathrm{pH}$ & 0.0194 & 0.0004 & Weak +ve \\
$\mathrm{EC}$ & -0.332 & 0.1102 & $-\mathrm{ve}$ \\
Clay & -0.2685 & 0.0721 & -ve \\
Silt & -0.1444 & 0.0209 & -ve \\
Sand & 0.3205 & 0.1027 & $+\mathrm{ve}$ \\
$\mathrm{SP}$ & -0.2621 & 0.0687 & $-\mathrm{ve}$ \\
$\mathrm{Na}$ & -0.3722 & 0.1385 & $-\mathrm{ve}$ \\
$\mathrm{K}$ & -0.5597 & 0.3133 & -ve \\
$\mathrm{P}$ & -0.6273 & 0.3935 & Moderate-ve \\
$\mathrm{Ca}$ & -0.6766 & 0.4578 & Moderate $-\mathrm{ve}$ \\
$\mathrm{Mg}$ & -0.577 & 0.3329 & Moderate $-\mathrm{ve}$ \\
$\mathrm{N}$ & -0.2104 & 0.0443 & Weak $-\mathrm{ve}$ \\
$\mathrm{O} . \mathrm{C}$ & -0.3295 & 0.1086 & Weak $-\mathrm{ve}$ \\
\hline
\end{tabular}

Table 7:Correlation Coefficients of the Physico-Chemical Properties with the Viable Bacterial Count (Cfu G ${ }^{-1}$ Soil) in AL-Aelafoon Sub-Region

\begin{tabular}{llll}
\hline Soil Physico-chemical Properties & $\mathrm{R}$ & $\mathrm{R}^{2}$ & Correlation \\
\hline $\mathrm{pH}$ & -0.3094 & 0.0957 & -ve \\
$\mathrm{EC}$ & 0.1062 & 0.0113 & +ve \\
Clay & 0.3816 & 0.1456 & $+\mathrm{ve}$ \\
$\mathrm{Silt}$ & 0.1936 & 0.0375 & $+\mathrm{ve}$ \\
$\mathrm{Sand}$ & -0.4129 & 0.1705 & $-\mathrm{ve}$ \\
$\mathrm{SP}$ & 0.8302 & 0.6892 & Strong +ve \\
$\mathrm{Na}$ & -0.0014 & 0 & -ve \\
$\mathrm{K}$ & 0.6252 & 0.3909 & Moderate +ve \\
$\mathrm{P}$ & -0.0983 & 0.0097 & Weak -ve \\
$\mathrm{Ca}$ & 0.0015 & 0 & Weak +ve \\
$\mathrm{Mg}$ & 0.1556 & 0.0242 & Weak +ve \\
$\mathrm{N}$ & -0.1926 & 0.0371 & Weak -ve \\
$\mathrm{O} . \mathrm{C}$ & -0.3388 & 0.1148 & Weak -ve \\
\hline & & & \\
\hline
\end{tabular}

Table 8: Correlation coefficients of the physico-chemical properties with the viable bacterial count ( $\mathrm{cfu} \mathrm{g}^{-1}$ soil) in Um Dawan Ban sub-region

\begin{tabular}{llll}
\hline Soil Physico-chemical Properties & $\mathrm{R}$ & $\mathrm{R}^{2}$ & Correlation \\
\hline $\mathrm{pH}$ & -0.1219 & 0.0149 & -ve \\
$\mathrm{EC}$ & -0.2512 & 0.0631 & -ve \\
Clay & 0.2614 & 0.0683 & +ve \\
Silt & 0.0216 & 0.0005 & +ve \\
Sand & -0.1771 & 0.0314 & -ve \\
$\mathrm{SP}$ & 0.565 & 0.3192 & Moderate +ve \\
$\mathrm{Na}$ & -0.4126 & 0.1702 & -ve \\
$\mathrm{K}$ & 0.9645 & 0.9303 & Strong +ve \\
$\mathrm{P}$ & 0.0197 & 0.0004 & Weak +ve \\
$\mathrm{Ca}$ & 0.7377 & 0.5442 & Moderate +ve \\
$\mathrm{Mg}$ & 0.0267 & 0.0007 & Weak +ve \\
$\mathrm{N}$ & 0.5215 & 0.272 & Moderate +ve \\
$\mathrm{O} . \mathrm{C}$ & 0.3214 & 0.1033 & Weak +ve \\
\hline
\end{tabular}

\section{Conclusion}

Qualitative analysis of microorganisms isolated from the studied soil samples reveal a total of thirteen different species of bacteria, of which two are unidentified. The ten species are classified under Bacillus genus, and one species is classified under Micrococcus genus.

The quantitative analysis of the isolated microorganisms was also carried out by considering individual colonies as separate units (CFUs). The quantitative data on microbial population recorded in the present study was analysed using two diversity indices. Soil Actinomyces spp. and Streptomyces spp. were the most abundant microorganisms identified in the three habitats.

Current biotechnolgy research is needed for developing new microbial pesticides from these studied microorganisms.

\section{References}

[1] Hassan HI and EL-Kamali HH (2015). Effect of soil physicchemical properties and plant type on bacterial diversity in semiarid parts in Central Sudan. Part I. Omdurman North region. Open Access Library Journal, 2, e1863. Doi:http://dx.doi.org/10.4236/Oalib 1101863. 
[2] Pettersson M. (2004). Factors affecting rates of change in soil bacterial communities. Doctoral Thesis. Lund University. Sweden.

[3] Cheesbrough, M (1984). CultureMedia. In: Medical Laboratory Manual for Tropical Countries. Tropical Health Technology and Butterworth-Heineman. Cambridge. Vol. III.

[4] Cruickshank R. (1975). Medical Microbiology: A Guide to Diagnosis and control of infection. 1st ed. Edinburgh and London: E and S Livingston Ltd. P. 888

[5] Barrow GHandFeltham RKA (1993). Cowan and Steel s Manual for Identification of Medical Bacteria. Third edition. Cambridge University Press, Cambridge PP.331.

[6] Williams ST and Cross T (1971). Actinomycetes: In: Methods in Microbiology, Booth,C. (Eds.). Academic Press, London.

[7] Collins CH, lyne PM and Grange J. (1995). Collins and Layne s Microbiological Methods. Butterworth-Heinemann, London.

[8] Benson T. (2001). Microbiological Applications Laboratory Manual in General Microbiology.Eighth Edition. The McGraw-Hill.

[9] You KM and Park YK (2004). A new method for the selective isolation of Actinomycetes from soil. BiotechnolTechn. 10:541-546.

[10] Richards L.A. (Ed.). 1954. Diagnosis and Improvement of Saline and Alkali Soils. US Dept. Agr. Handbook 60 US Gov. Printing Office. Washington, DC.

[11] Moghimi AH, HamdanJ, Shamshuddin J, Samsuri AW and Abtahi A (2013). Physico- chemical properties and surface charge characteristics of arid soils in Southern Iran. Applied and Environmental Soil Science Vol.2013, Article ID 252861. http://dx.doi.org/10.1155/2013/252861.

[12] Subrahmanyam NS and SambamurtyAVSS (2000). Ecology. Narosa Publishing House. New Delhi, P.172.

[13] Pearson Correlation Coefficient Calculator.Available at web site: www.socscistatistics.com/tests/pearson.

[14] Ndaw SM, Gama-Rodrigues AC, Gama-Rodrigues EF,Sales KR and Rosado AS (2009). Relationship between bacterial diversity,microbial biomass, and litter quality in soils under different plant covers in northern Rio de Janeiro State , Brazil. Can.J.Microbiol 55(9):1089-1095.http://dx.doi.org/10.1139/W09-066. 